\title{
The identification of a subgroup of children with traumatic subarachnoid hemorrhage at low risk of neuroworsening
}

\author{
Cecilia L. Dalle Ore, BA, ${ }^{1}$ Robert C. Rennert, MD, ${ }^{1}$ Alexander J. Schupper, BA, ${ }^{1}$ \\ Brandon C. Gabel, MD, ${ }^{1}$ David Gonda, MD, ${ }^{1,2}$ Bradley Peterson, MD, ${ }^{3}$ Lawrence F. Marshall, MD, ${ }^{1}$ \\ Michael Levy, MD, PhD, ${ }^{1,2}$ and Hal S. Meltzer, MD ${ }^{1,2}$ \\ 'Department of Neurosurgery, University of California San Diego School of Medicine; and Divisions of ${ }^{2}$ Neurosurgery and \\ ${ }^{3}$ Pediatric Critical Care, Rady Children's Hospital, San Diego, California
}

\begin{abstract}
OBJECTIVE Pediatric traumatic subarachnoid hemorrhage (tSAH) often results in intensive care unit (ICU) admission, the performance of additional diagnostic studies, and ICU-level therapeutic interventions to identify and prevent episodes of neuroworsening.

METHODS Data prospectively collected in an institutionally specific trauma registry between 2006 and 2015 were supplemented with a retrospective chart review of children admitted with isolated traumatic subarachnoid hemorrhage (tSAH) and an admission Glasgow Coma Scale (GCS) score of 13-15. Risk of blunt cerebrovascular injury (BCVI) was calculated using the BCVI clinical prediction score.
\end{abstract}

RESULTS Three hundred seventeen of 10,395 pediatric trauma patients were admitted with $\mathrm{tSAH}$. Of the 317 patients with $\mathrm{tSAH}, 51$ children (16\%, 23 female, 28 male) were identified with isolated $\mathrm{tSAH}$ without midline shift on neuroimaging and a GCS score of 13-15 at presentation. The median patient age was 4 years (range 18 days to 15 years). Seven had modified Fisher grade $3 \mathrm{tSAH}$; the remainder had grade $1 \mathrm{tSAH}$. Twenty-six patients (51\%) had associated skull fractures; 4 involved the petrous temporal bone and 1 the carotid canal. Thirty-nine (76.5\%) were admitted to the ICU and $12(23.5 \%)$ to the surgical ward. Four had an elevated BCVI score. Eight underwent CT angiography; no vascular injuries were identified. Nine patients received an imaging-associated general anesthetic. Five received hypertonic saline in the ICU. Patients with a modified Fisher grade $1 \mathrm{tSAH}$ had a significantly shorter ICU stay as compared to modified Fisher grade 3 tSAH (1.1 vs 2.5 days, $p=0.029$ ). Neuroworsening was not observed in any child.

CONCLUSIONS Children with isolated tSAH without midline shift and a GCS score of 13-15 at presentation appear to have minimal risk of neuroworsening despite the findings in some children of skull fractures, elevated modified Fisher grade, and elevated BCVI score. In this subgroup of children with $\mathrm{tSAH}$, routine ICU-level care and additional diagnostic imaging may not be necessary for all patients. Children with modified Fisher grade $1 \mathrm{tSAH}$ may be particularly unlikely to require ICU-level admission. Benefits to identifying a subgroup of children at low risk of neuroworsening include improvement in healthcare efficiency as well as decreased utilization of unnecessary and potentially morbid interventions, including exposure to ionizing radiation and general anesthesia.

https://thejns.org/doi/abs/10.3171/2018.5.PEDS18140

KEYWORDS neuroworsening; pediatric; traumatic brain injury; isolated traumatic subarachnoid hemorrhage; trauma

$\mathrm{T}$ RAUMATIC subarachnoid hemorrhage (tSAH) occurs in almost $6 \%$ of patients with traumatic brain injury (TBI), ${ }^{21}$ and in as many as $30 \%-60 \%$ of patients with moderate to severe TBI, ${ }^{4,28}$ and is a common sequela of pediatric head injury. ${ }^{4,28}$ The presence of tSAH following neurotrauma has been linked to unfavorable outcomes, including severe disability and death, 4,16,27,28 and patients with tSAH are at theoretical risk for rebleeding, ischemic complications, and posttraumatic hydrocephalus. ${ }^{4}$ Due to concerns over neurological deterioration, pe-

ABBREVIATIONS BCVI = blunt cerebrovascular injury; $\mathrm{CTA}=\mathrm{CT}$ angiography; $\mathrm{GCS}=$ Glasgow Coma Scale; $\mathrm{GOS}$ = Glasgow Outcome Scale; $\mathrm{HTS}=$ hypertonic saline; $\mathrm{ICU}=$ intensive care unit; $\mathrm{LOC}=$ loss of consciousness; LOS = length of stay; $\mathrm{MOI}=$ mechanism of injury; TBI = traumatic brain injury; $\mathrm{tSAH}=$ traumatic subarachnoid hemorrhage.

SUBMITTED March 6, 2018. ACCEPTED May 21, 2018.

INCLUDE WHEN CITING Published online August 10, 2018; DOI: 10.3171/2018.5.PEDS18140. 
diatric patients with tSAH are often transferred to tertiary care centers, admitted to intensive care units (ICUs), ${ }^{28}$ and followed using serial imaging (with potential exposure to ionizing radiation and/or general anesthetic agents) in an attempt to identify and address potential impending neuroworsening. ${ }^{30}$

However, isolated tSAH, particularly in the context of associated mild TBI, may represent a relatively benign entity. Adult patients who present with a Glasgow Coma Scale (GCS) score of 13-15 and tSAH without other intracranial mass lesions or midline shift typically have good outcomes and are unlikely to require neurosurgical intervention. $7,10,11,15,24,25$ While it has been suggested that the presentation of SAH is similar in adult and pediatric patients, ${ }^{23}$ specific data regarding the outcomes and management of SAH in the pediatric population remain limited. ${ }^{31}$

Risk stratification of pediatric patients with tSAH could allow for the identification of those at low risk for neuroworsening, preventing potentially unnecessary ICU admissions with attendant associated interventions, limiting additional exposure to ionizing radiation and administration of anesthetic agents, and improving overall healthcare efficiencies. In this paper we present the clinical course and neurological outcomes of 51 consecutive pediatric patients with isolated tSAH and mild TBI (GCS score 13-15) identified in a prospectively collected database of 10,395 pediatric trauma patients admitted over a 10 -year period.

\section{Methods \\ Data Collection}

Data regarding all consecutive patients who required trauma team activation at Rady Children's Hospital were collected prospectively from 2006 to 2015 as part of an institutionally specific pediatric trauma registry. With Rady Children's Hospital Trauma Advisory Committee and IRB approval, a retrospective chart review was conducted of all trauma registry patients admitted during this time period with a diagnosis of tSAH.

\section{Data Analysis}

Isolated tSAH was defined as an SAH that occurred in the context of a TBI without concurrent epidural, subdural, intraparenchymal, or intraventricular hemorrhage. SAHs were classified using the modified Fisher scale based on imaging in the electronic medical record and final attending radiologist interpretation. ${ }^{14}$ Risk of blunt cerebrovascular injury (BCVI) was calculated using the BCVI clinical prediction score described in Ravindra et al. ${ }^{38}$ GCS score $\leq 8$ corresponded to 1 point, focal neurological deficit to 2 points, carotid canal fracture 2 points, petrous temporal bone fracture 3 points, and cerebral infarct on CT to 3 points. Patients with scores of greater than 3 out of a possible total 11 points were designated high risk for BCVI. Neuroworsening was defined as described in Morris et al. ${ }^{30}$ Outcomes were classified using the Glasgow Outcomes Scale (GOS) based on documentation at discharge or, if available, the patient's most recent postdischarge well-child or subspecialty clinic visit.

Statistical analysis was conducted using SPSS (version
TABLE 1. Comorbid neurological injuries in all patients presenting with tSAH $(n=317)$

\begin{tabular}{lc}
\hline \multicolumn{1}{c}{ Diagnosis } & No. (\%) \\
\hline Epidural hemorrhage & $15(4.7)$ \\
\hline Subdural hemorrhage & $131(41.3)$ \\
\hline Intraventricular hemorrhage & $32(10.1)$ \\
\hline Cerebral contusion & $58(18.3)$ \\
\hline Diffuse axonal injury & $16(5)$ \\
\hline Cerebral edema & $37(11.7)$ \\
\hline Skull fracture & $177(55.8)$ \\
\hline Skull fracture w/ tissue loss & $18(5.7)$ \\
\hline Cervical spine injury & $14(4.4)$ \\
\hline
\end{tabular}

Patients with concurrent neurological injuries other than skull fracture were excluded from further analysis.

24.0.0.0, IBM). Fisher exact tests and Mann-Whitney Utests were used to conduct bivariate analyses.

\section{Results}

There were 10,395 pediatric trauma activations during the study period: 4481 were evaluated for minimal, mild, moderate, or severe TBI and 317 (7.5\% of all TBI patients) were admitted with tSAH as at least one of their admission diagnoses (mean GCS score 11.29, median 15). Of the 317 patients with a diagnosis of tSAH, 99 patients (31.2\%) were transferred from another hospital. Many possessed concomitant neurological injuries (Table 1), and 55 patients (17.3\%) died during their admission. Two hundred two patients had a GCS score of 13 or higher at the time of admission (Fig. 1). All patients received a pediatric neurosurgical consultation.

\section{Isolated tSAH}

Overall, 51 patients (16\% of patients with tSAH) had isolated tSAH without other intracranial hemorrhages or midline shift (Table 2). All patients with isolated tSAH had a GCS score of 13 or higher at presentation. Of the patients with isolated tSAH, the median age was 4 years (range 18 days to 15 years). Twenty-three patients were female and 28 were male. The mean GCS score was 14.9 (median 15).

The majority of patients $(n=30)$ presented following falls from a height of less than 10 feet, followed by motor vehicle collisions $(n=12)$, fall from a bike, skateboard, or scooter ( $\mathrm{n}=7,2$ helmeted), and fall from a height from more than 10 feet $(n=1)$. Nine patients had a high-energy mechanism of injury (MOI), defined as fall from a height of greater than 10 feet, pedestrian versus auto, or highspeed motor vehicle collision. Nine patients had a loss of consciousness (LOC) at the time of injury. One patient complained of diplopia at time of presentation.

Twenty-six patients $(51 \%)$ with tSAH had associated skull fractures, 4 involving the petrous temporal bone and 1 the carotid canal. Fourteen patients received multiple CT scans during their admission, none of which demonstrated $\mathrm{SAH}$ progression. In total, 8 patients underwent $\mathrm{CT}$ angi- 


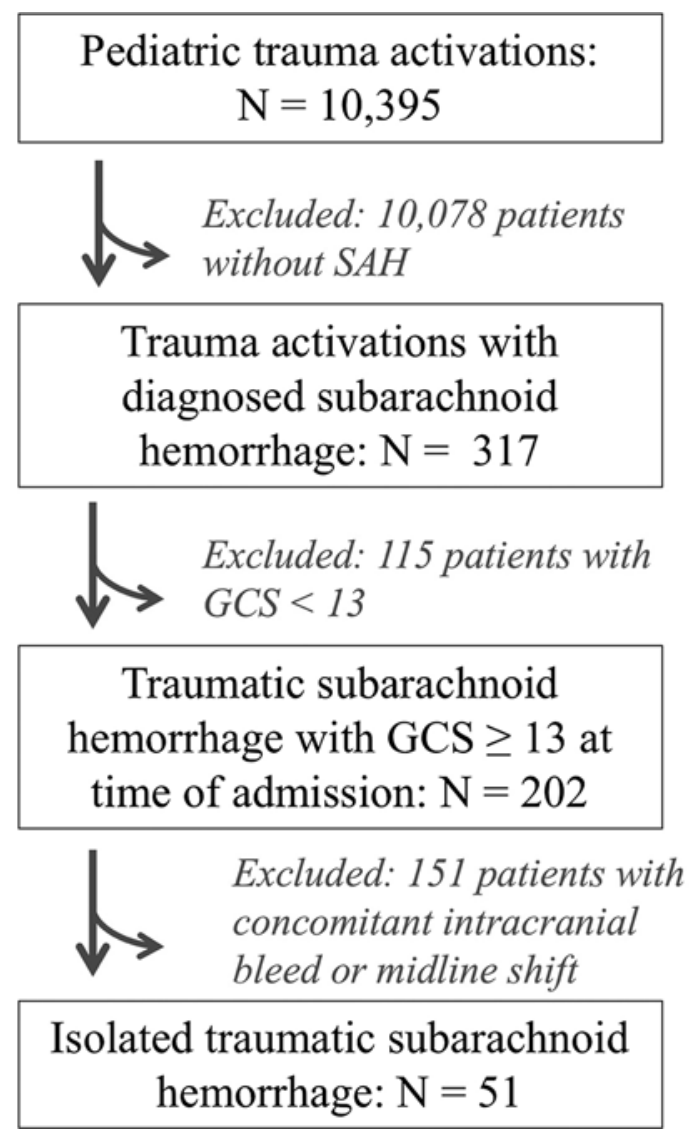

FIG. 1. Inclusion criteria for study analysis. Fifty-one patients were ultimately identified who met inclusion criteria, as defined by GCS scores of 13-15 and isolated $\mathrm{tSAH}$.

ography (CTA). Of the 8 patients who underwent CTA, 6 had modified Fisher grade 3 tSAH, 1 had modified Fisher grade $1 \mathrm{tSAH}$ with a skull base fracture involving the carotid canal (BCVI score of 5), and 1 patient had modified Fisher grade 1 tSAH with a compelling MOI (pedestrian vs auto). No vascular injuries were identified. Nine patients $(18 \%)$ who had imaging required a general anesthetic, 2 of whom had 2 anesthetics. At the time of presentation, 44 patients had modified Fisher grade 1 tSAH (Fig. 2), while the remaining $7(13.7 \%)$ with tSAH were modified Fisher grade 3 (Figs. 3 and 4; see Table 3 for characteristics of modified Fischer grade 1 and 3 patients).

On admission, patients with a modified Fisher grade $3 \mathrm{tSAH}$ had a mean GCS score of 14.7, as compared to a mean GCS score of 14.9 in patients with a modified Fisher grade 1 tSAH ( $p=0.030$; Table 3). Patients with modified Fisher grade 3 tSAH received a mean 2.85 head CT scans during their admission, while patients with modified Fisher grade $1 \mathrm{tSAH}$ received a mean of $1.15(\mathrm{p}<0.001)$, and $85.7 \%$ of patients with a Fisher grade 3 tSAH underwent CTA as compared to $4 \%$ of patients with a Fisher grade 1 tSAH ( $p<0.001)$. BCVI score was calculated retrospectively. Four patients in our cohort had an elevated BCVI score; of these patients, 2 had undergone CTA ( $p=0.111)$.
TABLE 2. Characteristics of patients with isolated tSAH

\begin{tabular}{lc}
\hline \multicolumn{1}{c}{ Variable } & Value \\
\hline No. of patients & 51 \\
\hline High-energy MOI & $9(17.6 \%)$ \\
\hline LOC & $9(17.6 \%)$ \\
\hline Mean admission GCS score (range) & $14.92(14-15)$ \\
\hline Multiple tSAHs & $8(15.7 \%)$ \\
\hline Skull fracture & $26(51.0 \%)$ \\
\hline BCVI $\geq 3$ & $4(7.8 \%)$ \\
\hline Mean no. of CT scans (range) & $1.39(1-5)$ \\
\hline CTA & $8(15.7 \%)$ \\
\hline ICU & $39(76.5 \%)$ \\
\hline Surgical floor & $12(23.5 \%)$ \\
\hline HTS & $5(9.8 \%)$ \\
\hline Mean ICU LOS (range), days & $1.29(0-4)$ \\
\hline Mean LOS (range), days & $1.84(1-11)$ \\
\hline Mean follow-up GOS score (range) & $4.9(4-5)$ \\
\hline
\end{tabular}

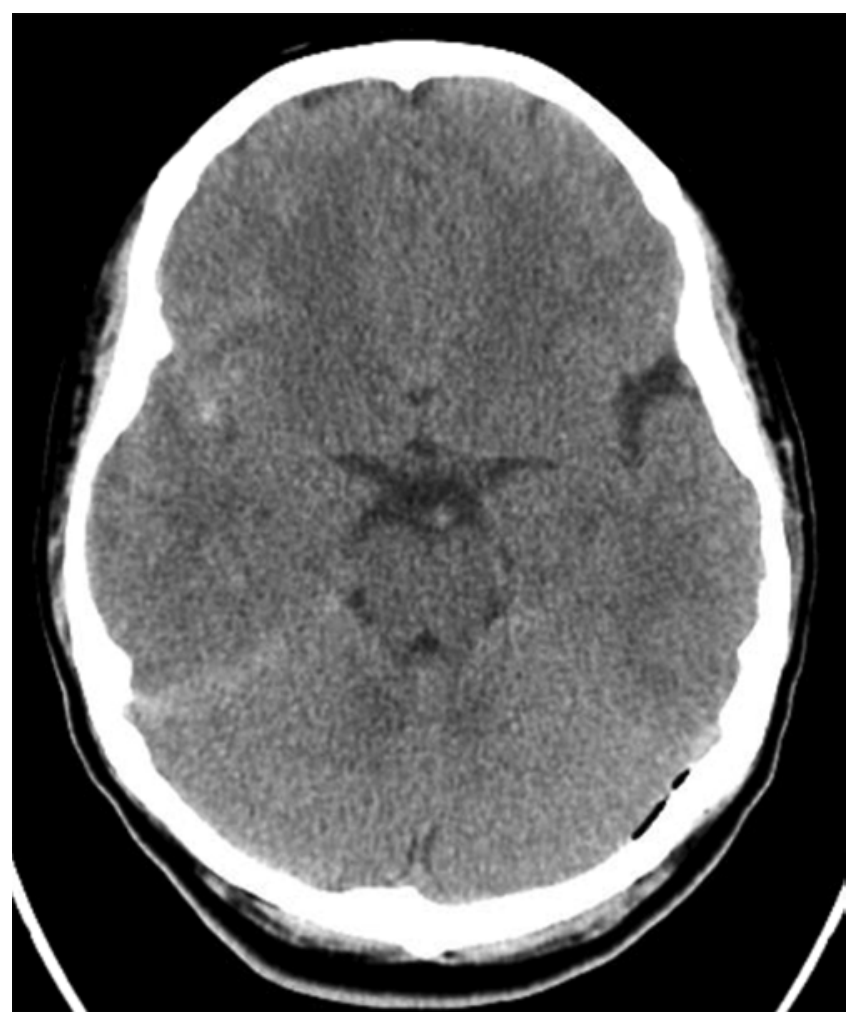

FIG. 2. Noncontrast head CT scan of a 9-year-old girl presenting following an MVC and demonstrating Fisher grade $1 \mathrm{tSAH}$. Patient GCS score was 15 at the time of admission. This patient was admitted to the pediatric ICU and received one bolus of 3\% HTS for headache. She was discharged directly from the ICU to home after 1 day following an uncomplicated hospitalization. At follow-up 3 years later she was at her neurological baseline with no residual deficits. 


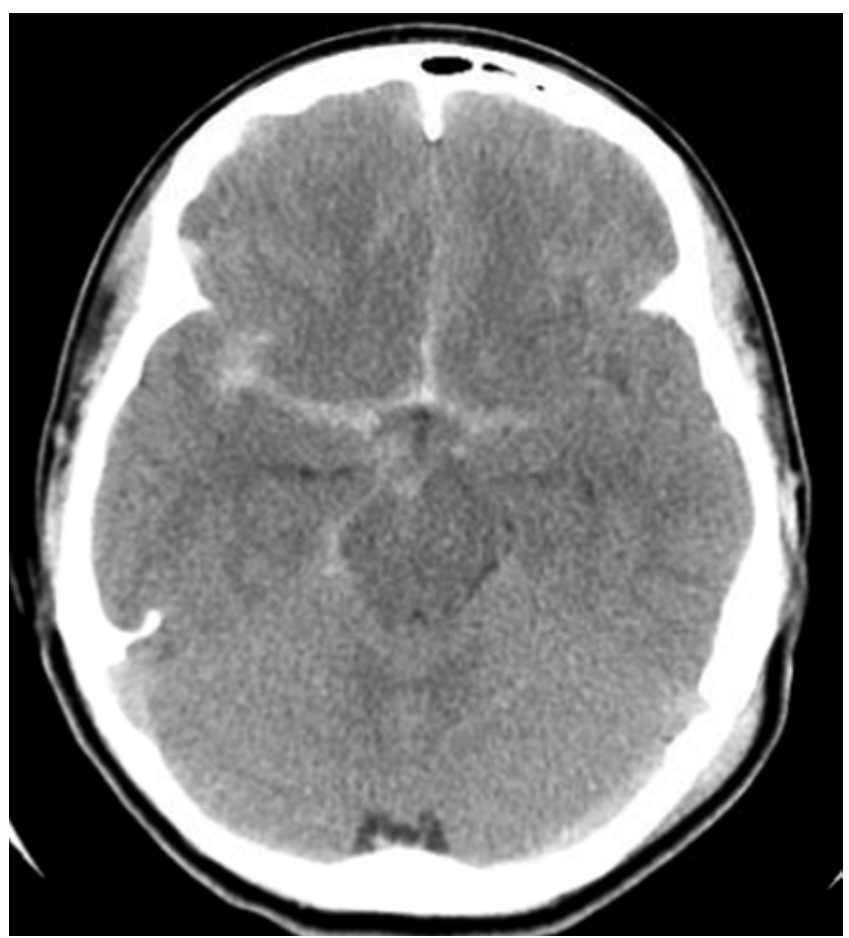

FIG. 3. Noncontrast CT head scan of a 14-year-old boy who presented following an unhelmeted skateboard fall, demonstrating modified Fisher grade $3 \mathrm{tSAH}$. At the time of presentation, the patient had a GCS score of 14 that improved to 15 during his hospitalization. CTA was obtained and was negative. He was admitted to the ICU for 3 days and discharged home directly from the ICU. He required no ICU or neurosurgical interventions (including osmotic agents) during his admission. At neurosurgical follow-up 1 year later, the patient was at his neurological baseline with no residual deficits.

All patients with an elevated BCVI score had been admitted to the ICU for observation ( $\mathrm{p}=0.561)$. None of the patients with an elevated BCVI score were found to have a vascular injury.

Thirty-nine patients $(76.5 \%)$ were admitted to the ICU, $12(23.5 \%)$ to a standard surgical ward (Table 4$)$. The association of ICU admission with a higher energy MOI trended toward significance $(p=0.092)$. Neuroworsening as indicated by a decline of more than 1 GCS point, GCS score decrease to below 13, or emergence of a new focal neurological deficit was not observed in any patient. Five patients received hypertonic saline (HTS) in the ICU for symptomatic management of headache and/or emesis. There was no statistically significant association of HTS administration with modified Fisher score $(\mathrm{p}=0.133)$ or BCVI score $(\mathrm{p}=$ $0.347)$. No patients were intubated, received an arterial or central venous line, underwent external ventricular drain or intraparenchymal pressure monitor placement, or required neurosurgical intervention. Thirty-six patients $(92.3 \%$ of ICU patients) were discharged directly from the ICU. The median ICU length of stay (LOS) was 1 day (mean 1.84 days). The average hospital LOS for ICU patients was 77\% longer than patients treated on the ward (2.02 vs 1.25 days; see Table 4 for characteristics of patients admitted to the ICU versus the surgical ward).

The mean LOS for patients with a modified Fisher

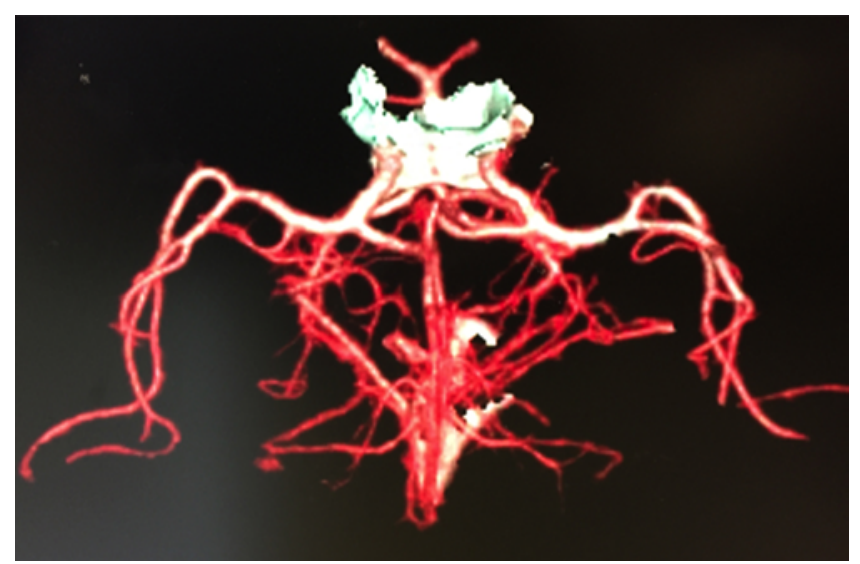

FIG. 4. CTA of the same patient with modified Fisher grade $3 \mathrm{tSAH}$ (Fig. 3) was negative for vascular injury. Figure is available in color online only.

grade 1 tSAH was 1.6 days (mean ICU stay of 1.1 days), while the mean LOS for patients with a Fisher grade 3 tSAH was 4.5 days (mean ICU stay of 2.5 days; overall LOS $p=0.099$, ICU LOS $p=0.029$ ). Ninety-six percent of patients with a Fisher grade $1 \mathrm{tSAH}$ admitted to the ICU were discharged directly from the ICU as compared to $71 \%$ of patients with a modified Fisher grade $3 \mathrm{tSAH}(\mathrm{p}$ $=0.077)$. All patients were discharged home.

One patient with a modified Fisher grade 3 tSAH was readmitted within 30 days of discharge for lethargy and emesis concerning for possible vasospasm. MR angiography and CTA were obtained at readmission and revealed a likely small contusion adjacent to his initial area of tSAH that was only observed on MRI. No vasospasm was noted. The patient was treated with HTS, his symptoms resolved, and he was discharged home with no long-term sequelae. Overall, the presence of Fisher grade 3 tSAH was not noted to be significantly associated with an increased risk of readmission $(\mathrm{p}=0.14)$.

All patients with isolated tSAH and GCS score $\geq 13$ at the time of presentation survived their hospitalization. The GOS score was $\geq 4$ for all patients at the time of discharge and follow-up (median follow-up 2 years, 10 patients lost to follow-up), consistent with a good neurological outcome. There was no statistically significant difference in GOS score by modified Fisher scale or by BCVI score ( $\mathrm{p}$ $=0.382$ and $\mathrm{p}=0.553$, respectively).

\section{Discussion}

To our knowledge, this paper represents the first published series of pediatric patients with isolated tSAH. $\mathrm{SAH}$, spontaneous as well as posttraumatic, can be associated with significant morbidity and mortality., ${ }^{4,16,27,28}$ In pediatric patients, the presence of tSAH in TBI has been associated with increased injury severity and adverse discharge. ${ }^{22}$ Children with tSAH are therefore routinely admitted to intensive care units for frequent neuromonitoring. However, data in the adult population suggest that isolated tSAH may actually be a relatively benign clinical entity, $7,10,11,24$ even in high-risk populations such as the elderly and those taking anticoagulant medications..$^{15}$ 
TABLE 3. Comparison of patients by modified Fisher grade and BCVI score

\begin{tabular}{|c|c|c|c|c|c|c|}
\hline Variable & Fisher Grade 1 & Fisher Grade 3 & p Value & $B C V I<3$ & $B C V I \geq 3$ & \\
\hline No. of patients & 44 & 7 & & 47 & 4 & \\
\hline Fisher grade 3 & 0 & 7 & NA & $6(12.8 \%)$ & $1(25 \%)$ & 0.457 \\
\hline $\mathrm{BCVI} \geq 3$ & $3(6.8 \%)$ & $1(14.3 \%)$ & 0.457 & $0(0 \%)$ & $4(100 \%)$ & NA \\
\hline High-energy MOI & $8(18.2 \%)$ & $1(14.3 \%)$ & 1.000 & $8(17.0 \%)$ & $1(25 \%)$ & 0.560 \\
\hline LOC & $6(13.6 \%)$ & $3(42.9 \%)$ & 0.079 & $8(17.0 \%)$ & $1(25 \%)$ & 0.560 \\
\hline Multiple tSAHs & $5(11.4 \%)$ & $3(42.9 \%)$ & 0.068 & $8(17.0 \%)$ & $0(0 \%)$ & 1.000 \\
\hline Skull fracture & $24(54.5 \%)$ & $2(28.6 \%)$ & 0.248 & $22(46.8 \%)$ & $4(100 \%)$ & 0.110 \\
\hline Mean GCS score (range) & $14.9(14-15)$ & $14.7(14-15)$ & 0.030 & $14.9(14-15)$ & $14.7(14-15)$ & 0.188 \\
\hline ICU & $32(72.7 \%)$ & $7(100 \%)$ & 0.117 & $35(74.5 \%)$ & $4(100 \%)$ & 0.561 \\
\hline Surgical floor & $12(27.3 \%)$ & $0(0 \%)$ & 0.117 & $12(25.5 \%)$ & $0(0 \%)$ & 0.561 \\
\hline CTA & $2(4.5 \%)$ & $6(85.7 \%)$ & $<0.001$ & $6(12.8 \%)$ & $2(50 \%)$ & 0.111 \\
\hline HTS & $3(6.8 \%)$ & $2(28.6 \%)$ & 0.133 & $4(8.5 \%)$ & $1(25 \%)$ & 0.347 \\
\hline Mean ICU LOS (range), days & $1.1(0-4)$ & $2.5(1-4)$ & 0.029 & $1.2(0-4)$ & $2.6(1-4)$ & 0.138 \\
\hline Mean LOS (range), days & $1.6(1-4)$ & $4.5(1-11)$ & 0.099 & $1.8(1-9)$ & $5(1-11)$ & 0.300 \\
\hline Mean GOS score (range) & $4.9(4-5)$ & $5(5)$ & 0.382 & $4.9(4-5)$ & $5(5)$ & 0.553 \\
\hline
\end{tabular}

This series reviews consecutive admissions over a 10year period from a large regional pediatric trauma referral center, which represents the pediatric neurotrauma patient population and current ICU/neurosurgical treatment paradigms for this region; $31.2 \%$ of children admitted with tSAH were transferred from other community medical facilities to our institution for definitive care. We identified 51 patients with isolated tSAH and mild TBI, and with a median age of 4 years, emphasizing the truly pediatric nature of this series.

We observed a $16.5 \%$ mortality rate among all patients with $\mathrm{tSAH}$, consistent with prior analyses identifying an association between nonisolated tSAH and significant risk

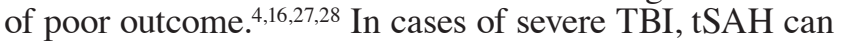
be indicative of a more severe MOI and may theoretically mediate further deleterious effects via inflammation and vasospasm. ${ }^{4}$ However, in contrast to these children at our institution, patients with isolated tSAH and mild TBI had no observed mortality or significant morbidity $(p<0.001)$. GOS score at the time of discharge (20\% of children) or at the most recent primary care or subspecialty clinic visit (80\% of children, median follow-up of 2 years) was $\geq 4$ and was not affected by either BCVI score or Fisher grade at the time of admission. While the 10 children who failed to attend their follow-up appointments all resided in the geographical referral base of our sole regional pediatric hospital/trauma center and would likely have returned had any significant postdischarge issue developed (as in the one patient who was readmitted), it is possible our reliance on GOS score at the time of discharge may have resulted in an underestimation of delayed worsening, particularly the potential development of subtle post-TBI cognitive issues. However, this would not impact our primary study endpoint of in-hospital episodes of neuroworsening, and

TABLE 4. Characteristics of patients admitted to the ICU versus the surgical ward

\begin{tabular}{lcccc}
\hline \multicolumn{1}{c}{ Variable } & Overall & ICU & Surgical Ward & p Value \\
\hline No. of patients & 51 & 39 & 12 & 0.092 \\
\hline High-energy MOI & $9(17.6 \%)$ & $9(23.1 \%)$ & 0 & 0.421 \\
\hline LOC & $9(17.6 \%)$ & $8(20.5 \%)$ & $1(8.3 \%)$ & 0.254 \\
\hline Mean admission GCS score (range) & $14.92(14-15)$ & $14.9(14-15)$ & $15(15)$ & 0.118 \\
\hline Mean modified Fisher grade (range) & $1.27(1-3)$ & $1.36(1-3)$ & $1(1)$ & 0.372 \\
\hline Multiple tSAHs & $8(15.7 \%)$ & $5(12.8 \%)$ & $3(25 \%)$ & 1.000 \\
\hline Skull fracture & $26(51.0 \%)$ & $20(51.3 \%)$ & $6(50 \%)$ & 0.561 \\
\hline BCVI $\geq 3$ & $4(7.8 \%)$ & $4(10.3 \%)$ & 0 & 0.088 \\
\hline Mean no. of CT scans & $1.39(1-5)$ & $1.48(1-5)$ & $1.08(1-2)$ & 0.173 \\
\hline CTA & $8(15.7 \%)$ & $8(20.5 \%)$ & 0 & 0.323 \\
\hline HTS & $5(9.8 \%)$ & $5(12.8 \%)$ & 0 & 0.202 \\
\hline Mean LOS (range), days & $1.84(1-11)$ & $2.02(1-11)$ & $1.25(1-2)$ & 0.200 \\
\hline Mean follow-up GOS score (range) & $4.9(4-5)$ & $4.86(4-5)$ & $5(5)$ & 0 \\
\hline
\end{tabular}


the overall excellent outcomes noted in our series suggest that, similar to findings in the adult population, pediatric isolated tSAH with mild TBI may represent a distinctly benign entity as compared to tSAH.

More than $50 \%$ of patients with isolated tSAH had a concurrent skull fracture, the majority of which did not involve the skull base. This finding is consistent with prior studies regarding pediatric TBI, which have reported a high incidence of skull fractures generally and a frequent association of tSAH with skull fractures. ${ }^{22,40}$ While skull fractures have been associated with an increased likelihood of contusion progression after TBI, in particular in combination with tSAH, ${ }^{2}$ pediatric patients with skull fractures and mild TBI typically have an excellent prognosis and are at a low risk for requiring ICU-level care. ${ }^{3}$

Typically, fractures of the skull base are associated with a more severe MOI, increased risk of acute adverse outcomes, and increased risk of BCVI. ${ }^{1,38,44} \mathrm{BCVI}$ is a potential vascular complication of nonpenetrating head injury, and may produce devastating neurological complications if not diagnosed and managed expeditiously.,45 Ravindra et al. developed a predictive model for identifying pediatric patients at increased risk for BCVI, and two of the significant factors identified were variants of skull base fractures, i.e., petrous temporal bone fractures and fractures through the carotid canal. ${ }^{38}$ The presence of a petrous temporal bone fracture alone was associated with a $32.4 \%$ probability of BCVI based on BCVI score, and patients with a fracture through the carotid canal had a $57.1 \%$ risk. The presence of either fracture designated the patient as high risk and additional imaging (i.e., CTA) was recommended. ${ }^{38}$ In a recent multicenter validation review of this predictive model, $9 \%$ of children screened with CTA after TBI were found to have a BCVI, more than $68 \%$ of whom required specific BCVI-related treatment. Additionally, almost $17 \%$ of the children screened with CTA had tSAH, highlighting the potential association of tSAH with a significant pediatric TBI-related vascular injury. ${ }^{37}$ In our series, 4 patients had fractures that involved the petrous portion of the temporal bone, one of which involved the carotid canal. This child as well as one of the other children with a petrous bone fracture had a CT angiogram, neither of which revealed a vascular injury. While CTA was negative in all 8 patients in this study of children with isolated tSAH, we would caution against drawing conclusions regarding any perceived lack of utility of CTA for potentially high-risk patients, such as those with elevated BCVI scores and/or petrous bone fractures, or children with high Fisher grade SAH. ${ }^{37}$ Given the previously reported potential for vascular injury associated with these characteristics, we recommend obtaining specific cerebrovascular imaging in this pediatric tSAH subgroup. ${ }^{37,38}$ Our practice has evolved over the course of this study and presently emphasizes BCVI score and modified Fisher grade in the determination of which patients warrant angiography, although we recognize additional patients may benefit from imaging based on their clinical picture. Further prospective tSAH studies regarding CTA and incorporating BCVI scoring would be of interest in this regard.

Morris et al. described neuroworsening as a potential endpoint of interest in patients with TBI and noted that one or more episodes of neuroworsening during the index admission for TBI was associated with poor outcomes. ${ }^{30}$ Further studies have corroborated these findings and demonstrated that neuroworsening is a poor prognostic factor associated with an increased risk of death and adverse discharge..$^{8,13,41}$ Episodes of neuroworsening mandate additional imaging and likely aggressive interventions to reduce associated neurological morbidity and serve as the rationale for increased level of care admissions such as ICU admission. ${ }^{13,20}$ Of the patients with isolated tSAH in this series, $76.5 \%$ were admitted to the ICU. However, no patients with tSAH (of any modified Fisher grade) experienced any episodes of neuroworsening or required invasive interventions.

Five of the children admitted to the ICU did receive HTS as part of their treatment regimen for headaches and/ or nausea (at the discretion of the trauma-ICU team), with improvement in overall symptomatology. Our institution initiated the treatment paradigms associated with the use of HTS in pediatric TBI and has also specifically described its potential utility in the treatment of postconcussive symptoms. ${ }^{26,33}$ However, we note that use of this intervention for postconcussive symptoms is not uniform, ${ }^{19,26}$ and certainly the majority of acute headaches in the pediatric mild TBI/ concussion population typically self-resolve over time. ${ }^{42}$

In the adult population, published rates of ICU admissions for isolated tSAH vary from $17 \%$ to $100 \%$, but it has been suggested that this elevated level of care may not be indicated for all patients in the context of mild TBI. ${ }^{24,25,34,35}$ In this series, more than $50 \%$ of patients with isolated tSAH admitted to the ICU were discharged from the ICU within 1 day (with more than $90 \%$ discharged directly home). These data suggest a potential overtriage regarding routine ICU admission.

The majority of patients $(86 \%)$ had modified Fisher grade $1 \mathrm{tSAH}$ at time of admission. The patients who presented with modified Fisher grade 3 tSAH received significantly more head CT scans ( $\mathrm{p}<0.001)$, were more likely to undergo CTA ( $\mathrm{p}<0.001)$, and had significantly longer ICU LOS $(p=0.029)$ as compared to those with Fisher grade $1 \mathrm{tSAH}$. Given the shorter admissions and reduced imaging utilization associated with modified Fisher grade $1 \mathrm{tSAH}$, these patients may be candidates for lower levels of observation.

Optimizing healthcare efficiencies is of increasing interest in regard to the delivery of care in the medical marketplace. ${ }^{9,18}$ According to a previously published analysis of healthcare costs in the US, the average cost difference between an ICU and a floor-level bed is approximately $\$ 2365$ per day, ${ }^{18}$ and the costs associated with ICU admission rose approximately $30 \%$ over one 5 -year period studied. ${ }^{18}$ Using this analysis for the 44 children with Fisher grade $1 \mathrm{tSAH}$ in our study and converting the mean ICU stay of 1.14 days to floor monitoring could represent a cost savings of $\$ 118,624$. Given that the first ICU day is associated with significantly higher costs than following days, limiting initial ICU admissions may produce even more significant cost savings than reduction in ICU LOS alone. ${ }^{17}$ Additionally, ICU admission has also been associated with adverse psychological sequelae for children and their families. ${ }^{36,43}$ Therefore, reducing unnecessary ICU admissions 
could potentially improve overall patient outcome, and patient and family satisfaction, as well as positively impact overall healthcare efficiencies.

The identification of isolated tSAH in children with GCS scores $\geq 13$ as a relatively benign clinical entity could also decrease the need for additional advanced imaging studies. Exposure to ionizing radiation and anesthetic administration has been associated with increased risk of cancer and developmental delay in children, respectively. ${ }^{29,32}$ Of the $27 \%$ of patients with isolated tSAH and mild TBI in this series who underwent repeat CT scans during their hospitalization, no patient demonstrated progression of their hemorrhage or underwent medical or surgical intervention as a result of imaging findings. This is consistent with multiple previous studies in both adult and pediatric patients discouraging the use of potentially unnecessary repeat head CT scans in TBI patients with an expected benign clinical course..$^{5,12,24,39}$ The data in this series suggest that routine repeat head CT scans may not be warranted in this population, especially in children with modified Fisher grade $1 \mathrm{tSAH}$ and no features placing them at a higher risk for BCVI.

Lastly, we note that this is a retrospective and observational study based on a prospectively collected trauma database regarding a specific subpopulation of pediatric patients with tSAH treated at a single major pediatric medical center. Clinical management practices such as ICU and surgical ward admission criteria and level of care, imaging interpretation, and anesthetic use may vary between centers; our results are most generalizable to similar institutions with extensive experience in the evaluation and critical care of pediatric trauma patients.

\section{Conclusions}

In this series, children with isolated tSAH without midline shift and GCS score of 13-15 at presentation were found to have minimal risk of neuroworsening, despite the findings in some children of vertex and basilar skull fractures, elevated modified Fisher grade, and elevated BCVI score. In this subgroup of children with tSAH, ICU-level observation and routine repeat diagnostic imaging may not be necessary. Patients with modified Fisher grade $1 \mathrm{tSAH}$ had a shorter LOS and were more likely to be discharged directly home from the ICU; these patients may be particularly unlikely to require ICU-level admission. Potential benefits to identifying a subgroup of children at low risk of neuroworsening could also include decreased exposure to ionizing radiation and general anesthesia in addition to overall optimization of healthcare efficiencies. While this study suggests that ICU admission, screening with CTA, and routine repeat neuroimaging may not be necessary for all children with isolated tSAH, clinical evaluation and risk assessment of individual patients should remain the foundation for making appropriate triage and overall management decisions.

\section{References}

1. Alhelali I, Stewart TC, Foster J, Alharfi IM, Ranger A, Daoud H, et al: Basal skull fractures are associated with mortality in pediatric severe traumatic brain injury. J Trauma Acute Care Surg 78:1155-1161, 2015
2. Allison RZ, Nakagawa K, Hayashi M, Donovan DJ, Koenig MA: Derivation of a predictive score for hemorrhagic progression of cerebral contusions in moderate and severe traumatic brain injury. Neurocrit Care 26:80-86, 2017

3. Ament JD, Greenan KN, Tertulien P, Galante JM, Nishijima DK, Zwienenberg M: Medical necessity of routine admission of children with mild traumatic brain injury to the intensive care unit. J Neurosurg Pediatr 19:668-674, 2017

4. Armin SS, Colohan AR, Zhang JH: Traumatic subarachnoid hemorrhage: our current understanding and its evolution over the past half century. Neurol Res 28:445-452, 2006

5. Brown CV, Zada G, Salim A, Inaba K, Kasotakis G, Hadjizacharia P, et al: Indications for routine repeat head computed tomography (CT) stratified by severity of traumatic brain injury. J Trauma 62:1339-1345, 2007

6. Carrillo EH, Osborne DL, Spain DA, Miller FB, Senler SO, Richardson JD: Blunt carotid artery injuries: difficulties with the diagnosis prior to neurologic event. J Trauma 46:11201125,1999

7. Chieregato A, Fainardi E, Morselli-Labate AM, Antonelli V, Compagnone C, Targa L, et al: Factors associated with neurological outcome and lesion progression in traumatic subarachnoid hemorrhage patients. Neurosurgery 56:671-680, 2005

8. Compagnone C, d'Avella D, Servadei F, Angileri FF, Brambilla G, Conti C, et al: Patients with moderate head injury: a prospective multicenter study of 315 patients. Neurosurgery 64:690-697, 2009

9. Coopersmith CM, Wunsch H, Fink MP, Linde-Zwirble WT, Olsen KM, Sommers MS, et al: A comparison of critical care research funding and the financial burden of critical illness in the United States. Crit Care Med 40:1072-1079, 2012

10. Deepika A, Munivenkatappa A, Devi BI, Shukla D: Does isolated traumatic subarachnoid hemorrhage affect outcome in patients with mild traumatic brain injury? J Head Trauma Rehabil 28:442-445, 2013

11. Ditty BJ, Omar NB, Foreman PM, Patel DM, Pritchard PR, Okor MO: The nonsurgical nature of patients with subarachnoid or intraparenchymal hemorrhage associated with mild traumatic brain injury. J Neurosurg 123:649-653, 2015

12. Durham SR, Liu KC, Selden NR: Utility of serial computed tomography imaging in pediatric patients with head trauma. J Neurosurg 105 (5 Suppl):365-369, 2006

13. Fan JS, Huang HH, Chen YC, Yen DH, Kao WF, Huang MS, et al: Emergency department neurologic deterioration in patients with spontaneous intracerebral hemorrhage: incidence, predictors, and prognostic significance. Acad Emerg Med 19:133-138, 2012

14. Frontera JA, Claassen J, Schmidt JM, Wartenberg KE, Temes $\mathrm{R}$, Connolly ES Jr, et al: Prediction of symptomatic vasospasm after subarachnoid hemorrhage: the modified Fisher scale. Neurosurgery 59:21-27, 2006

15. Gates M, Mallory G, Planchard R, Nothdurft G, Graffeo C, Atkinson J: Triage patterns of traumatic subarachnoid hemorrhage: is referral to a tertiary care center necessary? World Neurosurg 100:417-423, 2017

16. Greene KA, Marciano FF, Johnson BA, Jacobowitz R, Spetzler RF, Harrington TR: Impact of traumatic subarachnoid hemorrhage on outcome in nonpenetrating head injury. Part I: A proposed computerized tomography grading scale. J Neurosurg 83:445-452, 1995

17. Halpern NA: Can the costs of critical care be controlled? Curr Opin Crit Care 15:591-596, 2009

18. Halpern NA, Pastores SM: Critical care medicine in the United States 2000-2005: an analysis of bed numbers, occupancy rates, payer mix, and costs. Crit Care Med 38:65-71, 2010

19. Halstead ME: Pharmacologic therapies for pediatric concussions. Sports Health 8:50-52, 2016

20. Helling TS, Martin LC, Martin M, Mitchell ME: Failure 
events in transition of care for surgical patients. J Am Coll Surg 218:723-731, 2014

21. Heskestad B, Baardsen R, Helseth E, Romner B, Waterloo K, Ingebrigtsen T: Incidence of hospital referred head injuries in Norway: a population based survey from the Stavanger region. Scand J Trauma Resusc Emerg Med 17:6, 2009

22. Hochstadter E, Stewart TC, Alharfi IM, Ranger A, Fraser DD: Subarachnoid hemorrhage prevalence and its association with short-term outcome in pediatric severe traumatic brain injury. Neurocrit Care 21:505-513, 2014

23. Hourihan MD, Gates PC, McAllister VL: Subarachnoid hemorrhage in childhood and adolescence. J Neurosurg 60:1163-1166, 1984

24. Lee JJ, Segar DJ, Asaad WF: Comprehensive assessment of isolated traumatic subarachnoid hemorrhage. J Neurotrauma 31:595-609, 2014

25. Levy AS, Orlando A, Hawkes AP, Salottolo K, Mains CW, Bar-Or D: Should the management of isolated traumatic subarachnoid hemorrhage differ from concussion in the setting of mild traumatic brain injury? J Trauma 71:1199-1204, 2011

26. Lumba-Brown A, Harley J, Lucio S, Vaida F, Hilfiker M: Hypertonic saline as a therapy for pediatric concussive pain: a randomized controlled trial of symptom treatment in the emergency department. Pediatr Emerg Care 30:139-145, 2014

27. Maas AI, Hukkelhoven CW, Marshall LF, Steyerberg EW: Prediction of outcome in traumatic brain injury with computed tomographic characteristics: a comparison between the computed tomographic classification and combinations of computed tomographic predictors. Neurosurgery 57:11731182, 2005

28. Mattioli C, Beretta L, Gerevini S, Veglia F, Citerio G, Cormio M, et al: Traumatic subarachnoid hemorrhage on the computerized tomography scan obtained at admission: a multicenter assessment of the accuracy of diagnosis and the potential impact on patient outcome. J Neurosurg 98:37-42, 2003

29. Miglioretti DL, Johnson E, Williams A, Greenlee RT, Weinmann S, Solberg LI, et al: The use of computed tomography in pediatrics and the associated radiation exposure and estimated cancer risk. JAMA Pediatr 167:700-707, 2013

30. Morris GF, Juul N, Marshall SB, Benedict B, Marshall LF: Neurological deterioration as a potential alternative endpoint in human clinical trials of experimental pharmacological agents for treatment of severe traumatic brain injuries. Neurosurgery 43:1369-1374, 1998

31. Nahed BV, Ferreira M, Naunheim MR, Kahle KT, Proctor MR, Smith ER: Intracranial vasospasm with subsequent stroke after traumatic subarachnoid hemorrhage in a 22-month-old child. J Neurosurg Pediatr 3:311-315, 2009

32. Orser BA, Suresh S, Evers AS: SmartTots update regarding anesthetic neurotoxicity in the developing brain. Anesth Analg 126:1393-1396, 2018

33. Peterson B, Khanna S, Fisher B, Marshall L: Prolonged hypernatremia controls elevated intracranial pressure in headinjured pediatric patients. Crit Care Med 28:1136-1143, 2000

34. Phelan HA, Richter AA, Scott WW, Pruitt JH, Madden CJ, Rickert KL, et al: Does isolated traumatic subarachnoid hemorrhage merit a lower intensity level of observation than other traumatic brain injury? J Neurotrauma 31:1733-1736, 2014

35. Quigley MR, Chew BG, Swartz CE, Wilberger JE: The clinical significance of isolated traumatic subarachnoid hemorrhage. J Trauma Acute Care Surg 74:581-584, 2013
36. Ratzer M, Brink O, Knudsen L, Elklit A: Posttraumatic stress in intensive care unit survivors - a prospective study. Health Psychol Behav Med 2:882-898, 2014

37. Ravindra VM, Bollo RJ, Sivakumar W, Akbari H, Naftel RP, Limbrick DD Jr, et al: Predicting blunt cerebrovascular injury in pediatric trauma: validation of the "Utah Score". J Neurotrauma 34:391-399, 2017

38. Ravindra VM, Riva-Cambrin J, Sivakumar W, Metzger RR, Bollo RJ: Risk factors for traumatic blunt cerebrovascular injury diagnosed by computed tomography angiography in the pediatric population: a retrospective cohort study. J Neurosurg Pediatr 15:599-606, 2015

39. Rubino S, Zaman RA, Sturge CR, Fried JG, Desai A, Simmons NE, et al: Outpatient follow-up of nonoperative cerebral contusion and traumatic subarachnoid hemorrhage: does repeat head CT alter clinical decision-making? J Neurosurg 121:944-949, 2014

40. Sarkar K, Keachie K, Nguyen U, Muizelaar JP, ZwienenbergLee M, Shahlaie K: Computed tomography characteristics in pediatric versus adult traumatic brain injury. J Neurosurg Pediatr 13:307-314, 2014

41. Scheetz LJ, Horst MA, Arbour RB: Early neurological deterioration in older adults with traumatic brain injury. Int Emerg Nurs 37:29-34, 2018

42. Starkey NJ, Jones K, Case R, Theadom A, Barker-Collo S, Feigin V: Post-concussive symptoms after a mild traumatic brain injury during childhood and adolescence. Brain Inj 32:617-626, 2018

43. Sundararajan K, Martin M, Rajagopala S, Chapman MJ: Posttraumatic stress disorder in close Relatives of Intensive Care unit patients' Evaluation (PRICE) study. Aust Crit Care 27:183-187, 2014

44. Tunik MG, Powell EC, Mahajan P, Schunk JE, Jacobs E, Miskin M, et al: Clinical presentations and outcomes of children with basilar skull fractures after blunt head trauma. Ann Emerg Med 68:431-440, 440.e1, 2016

45. Weber CD, Lefering R, Kobbe P, Horst K, Pishnamaz M, Sellei RM, et al: Blunt cerebrovascular artery injury and stroke in severely injured patients: an international multicenter analysis. World J Surg 42:2043-2053, 2018

\section{Disclosures}

The authors report no conflict of interest concerning the materials or methods used in this study or the findings specified in this paper.

\section{Author Contributions}

Conception and design: Meltzer, Rennert, Gonda. Acquisition of data: Dalle Ore, Rennert, Schupper. Analysis and interpretation of data: Meltzer, Dalle Ore, Rennert, Schupper, Gabel, Gonda. Drafting the article: Meltzer, Dalle Ore, Rennert. Critically revising the article: Meltzer, Dalle Ore, Rennert, Gabel. Reviewed submitted version of manuscript: all authors. Approved the final version of the manuscript on behalf of all authors: Meltzer. Statistical analysis: Dalle Ore, Rennert. Study supervision: Meltzer.

\section{Correspondence}

Hal S. Meltzer: UCSD School of Medicine, Rady Children's Hospital, San Diego, CA. hsmeltzer@ucsd.edu. 